SAND98-0756 • UC-721

Unlimited Release

Printed March 1998

RECEIVED

MAY 271998

OSTI

\title{
A Suitable Low-Order, Eight-Node Tetrahedral Finite Element for Solids
}

S. W. Key, M. W. Heinstein, C. M. Stone, F. J. Mello, M. L. Blanford, K. G. Budge

Prepared by

Sandia National Laboratories

Albuquerque, New Mexico 87185 and Livermore, California 94550

Sandia is a multiprogram laboratory operated by Sandia Corporation,

a Lockheed Martin Company, for the United States Department of

Energy under Contract DE-AC04-94AL85000.

Approved for public release; further dissemination unlimited.

\section{Sandia National Laboratories}


Issued by Sandia National Laboratories, operated for the United States Department of Energy by Sandia Corporation.

NOTICE: This report was prepared as an account of work sponsored by an agency of the United States Government. Neither the United States Government nor any agency thereof, nor any of their employees, nor any of their contractors, subcontractors, or their employees, makes any warranty, express or implied, or assumes any legal liability or responsibility for the accuracy, completeness, or usefulness of any information, apparatus, product, or process disclosed, or represents that its use would not infringe privately owned rights. Reference herein to any specific commercial product, process, or service by trade name, trademark, manufacturer, or otherwise, does not necessarily constitute or imply its endorsement, recommendation, or favoring by the United States Government, any agency thereof, or any of their contractors or subcontractors. The views and opinions expressed herein do not necessarily state or reflect those of the United States Government, any agency thereof, or any of their contractors.

Printed in the United States of America. This report has been reproduced directly from the best available copy.

Available to DOE and DOE contractors from

Office of Scientific and Technical Information

P.O. Box 62

Oak Ridge, TN 37831

Prices available from (615) 576-8401, FTS 626-8401

Available to the public from

National Technical Information Service

U.S. Department of Commerce

5285 Port Royal Rd

Springfield, VA 22161

NTIS price codes

Printed copy: A03

Microfiche copy: A01

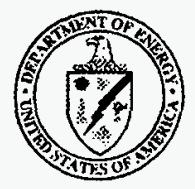


SAND98-0756

Distribution

Unlimited Release

Category UC-721

Printed March 1998

\title{
A Suitable Low-Order, Eight-Node Tetrahedral Finite Element For Solids
}

S. W. Key, M. W. Heinstein, C. M. Stone, F. J. Mello, M. L. Blanford Engineering and Manufacturing Mechanics Department

K. G. Budge

Computational Physics Research and Development Department

Sandia National Laboratories

P.O. Box 5800

Albuquerque, NM 87185-0443

\begin{abstract}
To use the all-tetrahedral mesh generation existing today, we have explored the creation of a computationally efficient eight-node tetrahedral finite element (a four-node tetrahedral finite element enriched with four mid-face nodal points). The derivation of the element's gradient operator, studies in obtaining a suitable mass lumping, and the element's performance in applications are presented. In particular we examine the eight-node tetrahedral finite element's behavior in longitudinal plane wave propagation, in transverse cylindrical wave propagation, and in simulating Taylor bar impacts. The element samples only constant strain states and,therefore, has 12 hourglass modes. In this regard it bears similarities to the eight-node, meanquadrature hexahedral finite element. Comparisons with the results obtained from the mean-quadrature ejght-node hexahedral finite element and the fournode tetrahedral finite element are included. Given automatic all-tetrahedral meshing, the eight-node, constant-strain tetrahedral finite element is a suitable replacement for the eight-node hexahedral finite element in those cases where mesh generation requires an inordinate amount of user intervention and direction to obtain acceptable mesh properties.
\end{abstract}




\section{Introduction}

Computer software designed for predicting the transient dynamic; large deformation, large strain, inelastic response of solids and structures based on the finite element method and explicit time integration requires fast, simple element formulations. That is, since it is expected in every calculation that the deformation will be finite and the material will be strained beyond the elastic range, the geometry and the associated gradient operators must be reconstructed, as well as complex stress-strain models evaluated at every step. As a result, finite elements derived for explicit transient dynamic software use the simplest and barest constructions possible for computational efficiency while retaining an essential representation of the physical behavior.

To date it has been possible to derive constant stress, eight-node hexahedral finite elements that satisfy the above expectations - for example, Flanagan and Belytschko [1981] and Flanagan and Taylor [1989]. Unfortunately, a tetrahedral finite element comparable to the constant stress, eight-node hexahedron does not yet exist. On paper the linear displacement, four-node tetrahedron appears to have the simplest and barest constructions possible for computational efficiency while retaining an essential representation of the physical behavior. It is, however, an abysmal performer for solids. Unfortunately, the linear displacement, four-node tetrahedral finite element continues to be used. (For extremely large deformations it is virtually impossible to turn the element inside out since it inust pass through a zero volume state. As a result, one always obtains numbers but rarely obtains meaningful answers.)

A recent Ph.D. thesis by Camacho, [1996] has a very careful exposition of the issues facing a developer seeking to find an improved tetrahedral element with capabilities that come close to what is available from a trilinear displacement, eight-node hexahedron.

Here we want to address transient dynamic applications, such as accident simulations of nuclear waste shipping containers and collateral damage estimation from accidental explosions as opposed to lower energy dynamic, quasi-static, or static simulations.

The approach that has been adapted here is to seek a minimally enriched linear displacement, four-node tetrahedral finite element as opposed, to say, a. minimally simplified quadratic displacement, 10-node tetrahedral finite element, $c f$. , Camacho [1996]. 


\section{Governing Equations}

\subsection{Motion}

The objective in a transient dynamic finite element program is to compute the motion of the body expressed in terms of displacement, velocity, and acceleration of every nodal point as a function of time for the entire simulation period. The theoretical development begins by formally introducing the concept of motion as a function that describes the position or configuration of the body at every instant in time.

A body $V$ is given that occupies a finite region of Euclidean space. Subjected to prescribed body forces and surface tractions, the body $I$ undergoes the motion $x^{i}=\chi^{i}\left(X^{\alpha}, t\right)$. The particles of the body are identified by the coordinates $X^{\alpha}$. They are referred to as material coordinates, and the relation of the particles to the coordinates $X^{\alpha}$ does not change in time. The places in space that the particles occupy during the motion are identified by the coordinates $x^{i}$, termed the spatial coordinates. The function $\chi^{i}$ describes the motion of the particles $X^{\alpha}$ through space as a function of time $t$. It is the motion $\chi^{i}$ that is sought.

The place occupied by the body at $t=0$ is taken as the reference configuration. In this configuration the body is assumed to be strain free, though not necessarily stress free. Only material coordinates $X^{\alpha}$ that coincide with the spatial coordinates $x^{i}$ in the reference configuration are considered. Thus, in the reference configuration $\chi^{i}\left(X^{\alpha}, 0\right) \equiv X^{\alpha}$.

While the material that follows can be considered to be expressed in terms of an arbilrary curvilinear coordinate system, it is sufficient to view the presentation as expressed in terms of a rectangular Cartesian coordinate system. Repeated upper and lower index pairs indicate a sum; lowrer case Latin and Greek indices have the range one to three, and upper case Latin indices (to appear later) range over the number of nodes in the element.

\subsection{Equations of Motion}

The Principle of Virtual Work provides the formal structure for developing the essential details of a finite element spatial discretization. In the terminology of functional analysis, the Principle of Virtual Work is a statement of the problem in the "weak" form. The terminology simply means that the Principle of Virtual Work admits solutions to the problem that are less 
smooth than are required by the differential equations ("strong" form). In particular the discontinuities in derivatives occurring at element boundaries are admissible in the Principle of Virtual Work.

Since the finite element method is, in fact, a means of constructing an approximate solution to the problem, it is instructive to clearly identify the differential equations represented by the Principle of Virtual Work. The Virtual Work expression is given by

$$
\delta \pi=\int_{V} \rho \ddot{x}^{k} \delta u_{k} d v+\int_{V} t^{k m} \delta u_{k, m} d v-\int_{V} \rho f^{k} \delta u_{k} d v-\oint_{S^{1}} s^{k} \delta u_{k} d a,
$$

and is required to vanish at all points along the path of motion for all variations $\delta u_{k}$ satisfying the displacement boundary conditions on $S^{2}$. The integration is performed over the current configuration of the body 1 , where $\rho$ is the mass density in that configuration, $\ddot{x}^{k}$ is the acceleration, $t^{k m}$ is the Cauchy stress - the stress in the current configuration, $f^{k}$ is the body force density in the current configuration, and $s^{k}$ is the surface traction that is acting on $S^{1}$. The comma denotes differentiation: $u_{i, j}=\partial u_{i} / \partial x^{j}$.

The divergence theorem is employed to reveal the differential equations of motion. In anticipation of using finite element approximations and the Galerkin method to generate approximate solutions, the case where $\delta u_{k, m}$ is only piecewise continuous is considered [Jones, 1964; Prager, 1967; and Key, 1971]. Interior surfaces, where the discontinuities of $\delta u_{k, m}$ occur, are denoted by $S^{0}$. Only surfaces $S^{0}$ that are stationary with respect to the material are considered. The situation is pictured in Figure 1 where $n_{k}$ is the normal to $S^{0}$ and the symbols + and - denote the respective sides of the surface. The result is

$$
\begin{aligned}
\int_{V}\left(\rho \ddot{x}^{k}-t_{, m}^{k m}-\rho f^{k}\right) \delta u_{k} d v & +\int_{S^{0}}\left(t_{+}^{k m}-t_{-}^{k m}\right) n_{m} \delta u_{k} d a \\
& +\oint_{S^{1}}\left(t^{k m} n_{m}-s^{k}\right) \delta u_{k} d a=0 .
\end{aligned}
$$

The differential form will vanish if and only if the integrand in each integral vanishes. The resulting expressions are the equations of motion,

$$
t_{, m}^{k m}+\rho f^{k}=\rho \ddot{x}^{k} \text { in } V
$$

the jump condition at a contact discontinuity,

$$
\left(t_{+}^{k m}-t_{-}^{k m}\right) n_{m}=0 \text { on } S^{0},
$$




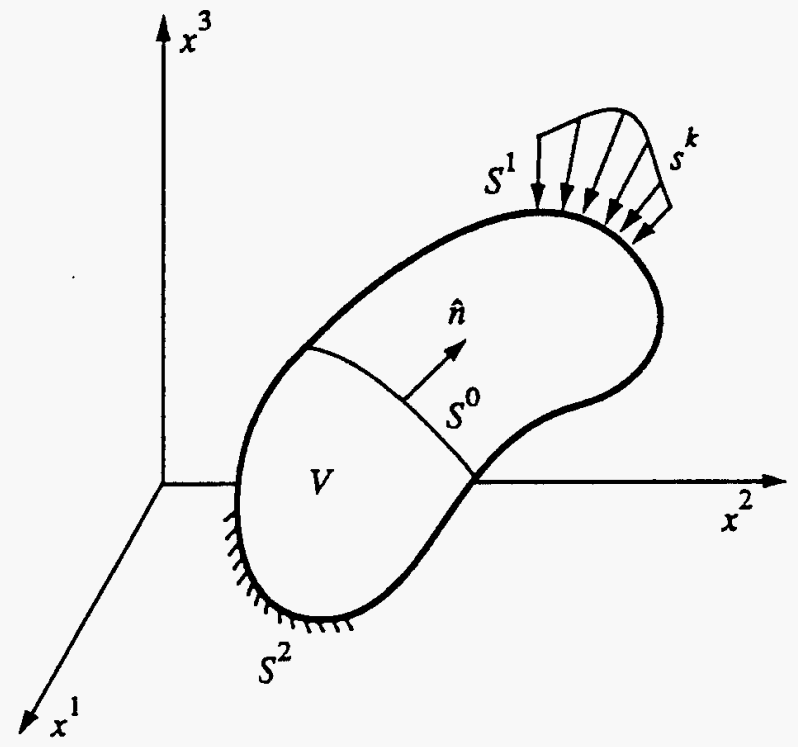

Figure 1: The body $I$ with surface traction $s^{k}$ on the boundary $S^{1}$ and a prescribed motion on the boundary $S^{2}$. An interior boundary $S^{0}$ with a unit normal vector $n^{k}$ is pictured.

and the traction boundary conditions,

$$
t^{k m} n_{m}=s^{k}(t) \text { on } S^{1} .
$$

Solutions obtained by using finite element approximations result in Equations 3,4 , and 5 being satisfied in an average sense - that is, within individual elements and along individual element boundaries these equations are satisfied in a mean or integral sense.

To Equations 3, 4, and 5 must be added the displacement boundary conditions on the surface $S^{2}$. These are called kinematic constraints and must be satisfied explicitly at each nodal point occurring on the surface $S^{2}$ by controlling the motion of the nodal points explicitly. The displacement boundary conditions are

$$
\chi^{i}\left(X^{\alpha}, t\right)=\kappa^{i}(t) \text { on } S^{2},
$$

where $\kappa^{i}$ prescribes the configuration of the boundary as a function of time. Only initial conditions that are homogeneous in position at time equal to 
zero are considered. Thus, the initial conditions are given by

$$
\begin{aligned}
& \chi^{i}\left(X^{\alpha}, 0\right)=X^{\alpha} \text { in } V, \\
& \dot{\chi}^{i}\left(X^{\alpha}, 0\right)=\nu^{i}\left(X^{\alpha}\right) \text { in } V,
\end{aligned}
$$

where $\nu^{i}$ prescribes the initial velocities at time equal to zero.

It is important to realize that these remarks are completely general with regard to the scale of deformation being considered. By using the current geometry - that is, the geometry as the body deforms - the Principle of Virtual Work assumes this very clean form. (This form of the Principle of Virtual Work may be contrasted with the form needed for static and quasi-static implementations where a configuration other than the current configuration must be used. The result is a series of transformations between the current configuration of the body and the geometry of the configuration used to develop the equations of motion. The attendant statement of the Principle of Virtual Work while exactly the same condition mathematically as used here, is much more complicated on paper and in code due to the explicit presence of the transformations.)

Again when the finite element Galerkin method is used to generate approximate solutions to Equation 1, it is Equations 3, 4, and 5 to which approximate solutions will be generated. Equation 6, the boundary condition on displacements, must be satisfied explicitly by the finite element Galerkin functions.

\section{Tetrahedral Finite Elements}

\subsection{Gradient/Divergence Operator}

This section provides the foundation for the subsequent development of the gradient/divergence operator for the proposed eight-node tetrahedron. The approach adapted for developing a mean strain rate quadrature for the eightnode tetrahedron is that given by Flanagan [1981] and Flanagan and Belytschko [1981]. While an initial reaction might be that the approach and notation of Flanagan are cumbersome, they provide the structure needed to achieve a closed-form solution for the integration of an arbitrary hexahedron in particular and, equally important, an explicit and unambiguous identification of the orthogonal hourglass modes that span the improper null space of the mean strain rate quadrature. 
The approach of Flanagan possesses a high degree of generality that becomes evident when additional finite elements are considered whether based on a constant stress state or higher-order variation in stress, $c f .$, Key and Hoff [1995]. Elements generated by this approach result in a de facto satisfaction of the first-order Irons patch test, provided a linearly varying motion can be represented exactly by the displacement assumptions within the element domain. With only minor modifications the material in this section is based on the work of Flanagan [1981].

Kinematics. Solid finite elements relate the spatial coordinates $x^{i}$ to the nodal coordinates $x_{I}^{i}$ through isoparametric shape functions $N^{I}$ as follows:

$$
x^{i}=x_{I}^{i} N^{I}\left(\xi^{i}\right) .
$$

In accordance with index notation convention, repeated subscript-superscript pairs imply summation over the range of that pair. The lower case subscripts and superscripts have a range of three, representing the spatial coordinate directions. Upper case subscripts and superscripts have a range that corresponds to the number of element nodal points.

The same shape functions are used to define the element displacement field in terms of the nodal displacements $u_{i l}$,

$$
u_{i}=u_{i I} N^{I}\left(\xi^{i}\right)
$$

Since these shape functions apply to both spatial coordinates and displacements, their material derivative (represented by a superposed dot) must vanish. Hence, the relocity field is given by

$$
v_{i}=v_{i I} N^{I}\left(\xi^{i}\right)
$$

The velocity gradient $v_{i, j}$ is defined as follows:

$$
v_{i, j}=v_{i I} N_{, j}^{I} \text {. }
$$

By convention a comma preceding a lower case subscript denotes differentiation with respect to the spatial coordinates, hence, $v_{i, j}$ denotes $\partial v_{i} / \partial x^{j}$.

As will be seen below, we do not need to make the form of the shape functions $N^{I}\left(\xi^{i}\right)$ explicit; we only need them conceptually to obtain a fundamental result relating the computation of a gradient/divergence operator to taking a derivative of the finite element's volume. 
Mean Strain Rate Quadrature. The Principle of Virtual Work gives the following relationship for the element nodal forces $f^{i l}$ due to the divergence of the stress field,

$$
v_{i I} f^{i I}=\int_{1} t^{i j} d_{i j} d v
$$

Since the Cauchy stress tensor $t^{i j}$ (force per unit area in the current configuration) is symmetric, the velocity gradient may replace the stretching tensor, $d_{i j}=v_{(i, j)}$, above. To obtain an explicit representation of the nodal forces $f^{i I}$, one must introduce a finite element. The objective of the material to follow is a rigorous development of the explicit expressions for computing $f^{i I}$ from the motion.

The integral in Equation 12 is evaluated using a constant stress, thereby considering only a mean strain rate within the element. 'The preceding expression is approximated by

$$
v_{i I} f^{i I}=V \bar{t}^{i j} \bar{i}_{i, j}
$$

The assumed constant stress field is represented by $\bar{t}^{i j}$, which will be referred to as the mean stress tensor. It is assumed that the mean stress depends only on the mean strain. Mean kinematic quantities are defined by integrating over the element as follows:

$$
\bar{v}_{i, j}=(1 / V) \int_{V} v_{i, j} d v .
$$

The gradient operator $B_{i}^{I}$ is defined by

$$
B_{i}^{I} \equiv \int_{v} N_{, i}^{I} d v
$$

The mean velocity gradient, applying Equation 15, is then given by

$$
\bar{v}_{i, j}=(1 / V) v_{i I} B_{j}^{I} .
$$

The nodal forces are then given by the divergence operation,

$$
f^{i I}=\bar{t}^{i j} B_{j}^{I} \text {. }
$$

Computing nodal forces by this integration scheme requires evaluation of the gradient operator $B_{i}^{I}$ and volume. These two tasks can be linked by using $x_{, j}^{i}=\delta_{j}^{i}$, which when used in Equation 15 yields

$$
x_{I}^{i} B_{j}^{I}=\int_{V}\left(x_{I}^{i} N^{I}\right), j d v=V \delta_{j}^{i} .
$$


Consequently; the gradient operator $B_{i}^{I}$ may alternatively be expressed by

$$
B_{i}^{I}=\frac{\partial V}{\partial x_{I}^{i}} .
$$

Thus, obtaining a mean gradient/divergence operator $B_{i}^{I}$ has been reduced to finding the volume of an element $V\left(x_{I}^{i}\right)$ in closed form.

Remark: There is a consistency requirement that the shape functions $N^{I}$ must satisfy for the above derivation to hold - namely, $b_{I} N^{I}$ must be able to reproduce exactly a linear function where the $b_{I}$ are constants.

\subsection{Four-Node Tetrahedral Element}

For a four-node linear tetrahedron, the element volume in closed form is easily constructed from vector operations with its edges,

$$
V=\int_{1} d v=\epsilon_{i j k}\left(x_{4}^{i}-x_{1}^{i}\right)\left(x_{2}^{j}-x_{1}^{j}\right)\left(x_{3}^{k}-x_{1}^{k}\right) / 6 .
$$

As a result, the gradient operator for the four-node tetrahedron is given by

$$
\begin{aligned}
B_{x}^{1} & =\frac{1}{6}\left(y_{34} z_{24}-y_{24} z_{34}\right) \\
B_{x}^{2} & =\frac{1}{6}\left(y_{13} z_{14}-y_{14} z_{13}\right) \\
B_{x}^{3} & =\frac{1}{6}\left(y_{14} z_{12}-y_{12} z_{14}\right) \\
B_{x}^{4} & =\frac{1}{6}\left(y_{12} z_{13}-y_{13} z_{12}\right)
\end{aligned}
$$

where $\left\{x_{I}^{i}\right\}=\left\{x_{I}, y_{I}, z_{I}\right\}$ and $z_{I J}=z_{I}-z_{J}$ et cetera. To obtain the components $B_{y}^{I}$ and $B_{z}^{I}$ the coordinate permutations contained in Table 1 are used.

As is well known the four-node linear tetrahedral element provides exceptionally poor computational results for solids. In the next section our purpose is to enrich the four-node tetrahedral element with the expectation of providing results comparable in quality and numerical efficiency to that obtained with the eight-node hexahedral elements extant today. 
Table 1:

Permutation of Coordinates for Use with Equation 21

\begin{tabular}{|c|c|c|c|c|c|}
\hline$B_{i}^{I}$ & Coordinates & $B_{i}^{I}$ & Coordinates & $B_{i}^{I}$ & Coordinates \\
\hline$B_{x}^{I}$ & $y \quad z$ & $B_{y}^{I}$ & $z$ & $B_{z}^{I}$ & $x$ \\
\hline
\end{tabular}

\subsection{Eight-Node Tetrahedral Finite Element}

The minimum number of additional degrees of freedom is obtained by adding a nodal point to each of the faces of a four-node tetrahedron. Considering a displacement of a face node, it can be observed that a displacement normal to the face will have a greater effect on the volume change of the element than on changes in shear distortion. Conversely, a displacement tangential to the face will have a greater effect on the shear distortion of the element than on a volume change. We defer until later the question of whether to use only the normal displacement at each face node, only the two tangential displacements at each face node, or all three degrees of freedom.

Ordinarily the addition of nodal points to a linear element engenders the introduction of quadratic terms in the shape functions. However, since our objective here is to use only the mean strain produced by movement of the element's nodal points, we have some liberty in how we relate deformation within the element interior to the movement of each nodal point.

Here we choose to use a linear "sub-tetrahedron" based on the three vertex nodal points of the face and the newly introduced mid-face nodal point, Figure 2. Thus, the volume of the eight-node enriched tetrahedron is given by the volume of the original or parent four-node tetrahedron $l_{0}$ and the volume changes introduced by movement of the mid-face nodes,

$$
\begin{aligned}
V & =V_{0}\left(x_{1}^{i}, x_{2}^{i}, x_{3}^{i}, x_{4}^{i}\right)+V_{1}\left(x_{1}^{i}, x_{2}^{i}, x_{4}^{i}, x_{7}^{i}\right) \\
& +V_{2}\left(x_{2}^{i}, x_{3}^{i}, x_{4}^{i}, x_{5}^{i}\right)+V_{3}\left(x_{3}^{i}, x_{1}^{i}, x_{4}^{i}, x_{6}^{i}\right) \\
& +V_{4}\left(x_{1}^{i}, x_{3}^{i}, x_{2}^{i}, x_{8}^{i}\right) .
\end{aligned}
$$

The mean gradient/divergence operator $B_{i}^{I}$ is given by

$$
B_{i}^{I}=\frac{\partial V_{0}}{\partial x_{I}^{i}}+\frac{\partial V_{1}^{i}}{\partial x_{I}^{i}}+\frac{\partial V_{2}}{\partial x_{I}^{i}}+\frac{\partial V_{3}}{\partial x_{I}^{i}}+\frac{\partial V_{i}}{\partial x_{I}^{i}}
$$




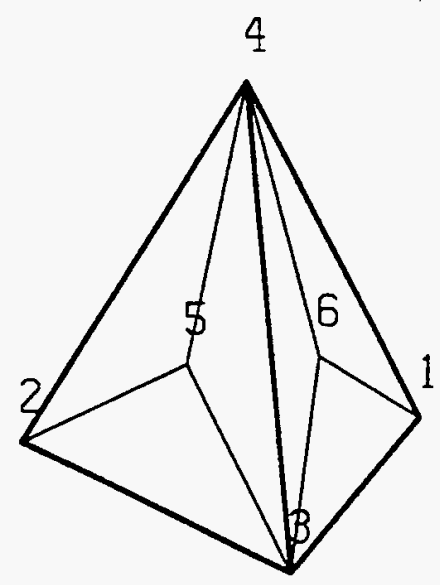

Figure 2: A linear four-node tetrahedron $(i, j, k, l=1,2,3,4)$ to which has been added four mid-face nodal points $(m, n, o, p=5,6,7,8)$. A linear "subtetrahedron" is associated with each mid-face nodal point so that - for example, $(j, k, l, m=2,3,4,5)$.

Since it is desirable to keep the element implementation and subsequent interpretation of results as straightforward as possible, we are going to retain all three degrees of freedom at each mid-face nodal point. Retention of all three degrees of freedom at the mid-face nodal points will allow the vertex nodes and the newly introduced mid-face nodal points to share, with maximum flexibility, the task of representing the shear or deviatoric part of the solution and the bulk part of the solution.

The mean gradient/divergence operator only describes six uniform strain states. Strain states with higher-order variation are ignored - that is, the eight-node tetrahedron is "under integrated." Because the element is under integrated, we are left with a number of hourglass modes - twelve to be precise.

At this point it is worthwhile to examine the stiffness properties of the eight-node mean-quadrature tetrahedral element. For comparison purposes the stiffness properties for three scparate, isolated tetrahedra - that is, three separate single finite elements will be examined. The equilateral tetrahedron shown in Figure 2 is used in turn to represent three different tetrahedral elements: (1) a fully integrated four-node linear tetrahedral element, (2) an eight-node mean-quadrature tetrahedral element, and (3) as it happens to work out, an assembly of 11 non-overlapping four-node linear sub-tetrahedra. 
(This ability to describe the eight-node mean-quadrature tetrahedron as an assembly of 11 , four-node linear tetrahedra is not only useful for examining the benefit of retaining just the 6 uniform strain states but allows a convenient representation of the shape functions. It is also possible to obtain the same eight-node mean-quadrature gradient/divergence operator of Equation $23 \mathrm{by}$ using a volume-weighted assembly of the operators belonging to the 11 fournode linear tetrahedral decomposition, although not as efficiently calculated.)

Table 2 shows the eigenvalues calculated for each of the three separate single-element stiffness matrices. Note that in the case of the eight-node mean-quadrature tetrahedron, the largest eigenvalue corresponds to a volumetric deformation eigenvector; and the five smaller, repcated eigenvalues correspond to constant pure-shear eigenvectors. The remaining 18 zero eigenvalues correspond either to the "hourglass" modes not supported by the mean quadrature or to the 6 rigid body modes.

One pure-bulk and five equal pure-shear eigenvalue-eigenvector pairs is the desired result. The separation into pure-bulk and pure-shear modes was confirmed by computing a Rayleigh quotient with each eigenvector paired first with an element stiffness matrix based only on the bulk modulus and second with an element stiffness matrix based only on the shear modulus.

We do not have an interpretation for the differences in eigenvalue magnitudes between the fully integrated four-node linear tetrahedral element and the eight-node mean-quadrature tetrahedral element.

\subsection{Lumped Mass Representation}

The eight-node mean quadrature tetrahedral element does not immediately suggest how the mass should be apportioned between the vertex nodal points and the mid-face nodal points. For dynamic simulations it is important to obtain the correct distribution of mass between the vertex nodal points and the mid-face nodal points. Both for speed and for accuracy, a lumped mass matrix is preferred over a consistent mass matrix when using an explicit central difference time integration scheme, Krieg and Key [1973].

Standard practice is to generate a consistent mass matrix first, and then perform a row sum to obtain the diagonal lumped mass matrix. For this task the shape functions represented by the 11 non-overlapping four-node linear tetrahedral decomposition are convenient. Since the shape functions are nonoverlapping across the individual subtetrahedra, each of the 11 individual mass matrices need only be calculated and assembled. Each individual mass 
Table 2:

Eigenvalues for Three Separate Tetrahedral Elements.

Young's modulus $=10^{\bar{T}}$, Poisson's ratio $=0.25$

\begin{tabular}{|c|c|c|}
\hline $\begin{array}{c}\text { Single } \\
\text { Four-Node } \\
\text { Tetrahedron }\end{array}$ & $\begin{array}{c}\text { Single } \\
\text { Eight-Node } \\
\text { Tetrahedron }\end{array}$ & $\begin{array}{c}\text { Assembly of } \\
\text { Four-Node } \\
\text { Tetrahedra }\end{array}$ \\
\hline $4.7138 \times 10^{6}$ & $6.8061 \times 10^{6}$ & $13.087 \times 10^{6}$ \\
$1.8855 \times 10^{6}$ & $2.7475 \times 10^{6}$ & $9.188 \times 10^{6}$ \\
$1.8855 \times 10^{6}$ & $2.7475 \times 10^{6}$ & $9.188 \times 10^{6}$ \\
$1.8855 \times 10^{6}$ & $2.7475 \times 10^{6}$ & $8.231 \times 10^{6}$ \\
$1.8855 \times 10^{6}$ & $2.7475 \times 10^{6}$ & $8.231 \times 10^{6}$ \\
$1.8855 \times 10^{6}$ & $2.7475 \times 10^{6}$ & $4.843 \times 10^{6}$ \\
0 & 0 & $4.843 \times 10^{6}$ \\
$\vdots$ & $\vdots$ & $\vdots$ \\
0 & 0 & 0 \\
\hline
\end{tabular}

matrix is computed with the formula, Hughes [1987],

$$
m_{i j}=\int_{V} \rho N_{i} N_{j} d v
$$

and has the form

$$
\left[m_{i j}\right]=\frac{\rho V}{20}\left[\begin{array}{llll}
2 & 1 & 1 & 1 \\
1 & 2 & 1 & 1 \\
1 & 1 & 2 & 1 \\
1 & 1 & 1 & 2
\end{array}\right]
$$

The lumped mass matrix for the eight-node mean-quadrature tetrahedral element resulting from this approach places 11/108 of the total mass at each vertex nodal point and $16 / 108$ of the total mass at each mid-face nodal point.

Unfortunately; the dynamic performance of the eight-node mean-quadrature tetrahedral element with this mass lumping is not what it should be. We have resorted to considering various combinations of vertex and mid-face nodal point mass distributions. Shown in Table 3 is a number of the masslumping variants examined together with the six eigenvalues, $(k-\lambda m) \phi=0$, 
Table 3:

Eigenvalues $\left(\times 10^{8}\right)$ for Various Mass Representations.

Column headings: vertex weighting $\&$ mid-face weighting

\begin{tabular}{|c||c|c|c|c||c|}
\hline Consistent & $\frac{11}{108} \& \frac{16}{108}$ & $\frac{1}{6.7} \& \frac{1}{10}$ & $\frac{1}{6} \& \frac{1}{12}$ & $\frac{1}{5} \& \frac{1}{20}$ & 4-Node Tet \\
\hline 8.4599 & 4.4402 & 5.1774 & 5.8572 & 8.8730 & 1.5985 \\
3.8515 & 1.7765 & 2.0715 & 2.3434 & 3.5500 & 0.6396 \\
3.8515 & 1.7766 & 2.0713 & 2.3432 & 3.5497 & 0.6395 \\
2.9712 & 1.7762 & 2.0711 & 2.3431 & 3.5496 & 0.6394 \\
2.9712 & 1.7756 & 2.0705 & 2.3423 & 3.5484 & 0.6392 \\
2.3804 & 1.7756 & 2.0705 & 2.3423 & 3.5484 & 0.6392 \\
\hline
\end{tabular}

obtained from using them with the equilateral tetrahedron shown in Figure 2. In addition, the separation into pure-bulk and pure-shear modes was confirmed by computing a Rayleigh quotient with each mode shape paired first with an element stiffness matrix based only on the bulk modulus and second with an element stiffness matrix based only on the shear modulus. It is seen that with any of the mass lumping schemes, the desired property of having the response represented by one bulk deformation mode and five equal-energy shear deformation modes is independent of the mass lumping. The eigenvalues by themselves do not suggest an apportionment between the vertex nodal points and the mid-face nodal points either.

The most rational criteria for mass lumping we have been able to generate is to examine the results from wave propagation simulations. In doing so two positive results occurred simultaneously: (1) the correct stress wave arrival time occurred in the vertex nodal points and mid-face nodal points when the mass lumping was $1 / 6$ and $1 / 12$, respectively, and (2) excitation of the hourglass modes due to the passage of the stress wave was near zero for this mass lumping.

Using a constant pressure instantaneously applied on one end of a bar that is constrained to have no lateral molion produces a planar longitudinal wave propagating down the bar. By selecting a cross section and examining the arrival time, the quality of the mass lumping at the nodal points can be 
evaluated. Figure 3 shows the axial velocity history of an elastic wave as it arrives at the middle of the bar. The arrival time is correct based on when the "mid-height" of the wave front reaches the nodal points on the cross section. The planarity of the wave is shown by how closely the individual nodal point responses match. (No artificial bulk viscosity was used with the explicit central difference time integration algorithm in order to enhance any differences in behavior between the vertex and mid-face nodal points.)

Figure 4 shows the bulk, deviatoric, and hourglass strain energy (corrected for arrival time) in each of six elements constituting a unit cell at the middle of the bar. (The hourglass strain energy is multiplied by 100 and is the strain energy that would occur if the hourglass deformation were to be applied to an eight-node tetrahedron obtained by assembling 11, fournode linear tetrahedral elements, a "fully integrated" eight-node tetrahedral element.)

To assess in what way the mid-face nodal points serve to increase the elements capacity to represent the deformation, a Rayleigh quotient was computed using the "incremental" deformation provided by the mid-face nodal points paired first with an element stiffness matrix based only on the bulk modulus and second with an element stiffness matrix based only on the shear modulus. The result is a nearly equal contribution to the bulk strain energy and to the deviatoric strain energy. (By "incremental" deformation we mean the additional deformation provided by a mid-face nodal point beyond that movement implied by the bounding vertices - for example, with reference to Figure 2, $\Delta u_{5}^{i}=u_{5}^{i}-\left(u_{2}^{i}+u_{3}^{i}+u_{4}^{i}\right) / 3$.)

\subsection{Surface Traction}

A satisfactory representation of surface traction as equivalent nodal loads is a prescription needed to complete the element's implementation. Since the underlying shape functions are piecewise linear, the traction on each triangular surface facet is converted to a total force and distributed onethird to each nodal point. (For triangular facets consistent lumping results in one-third of the integrated surface pressure applied to each of the three vertex nodal points.) 


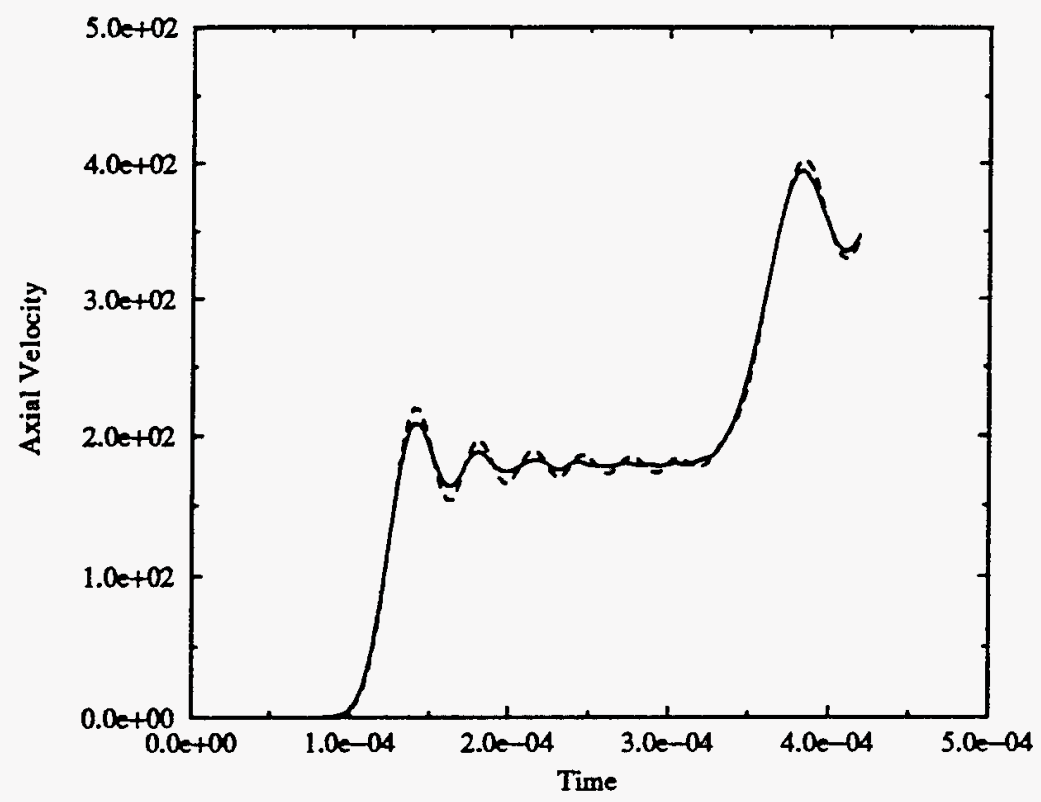

Figure 3: The axial velocity at a vertex (dashed curve) and mid-face (solid curve) nodal point in the middle of a bar 50 units long subjected to a step in pressure on one end and free on the other end. Young's modulus $=10^{7}$, Poisson's ratio $=0.25$, density $=2.61 \times 10^{-4}$. The mesh consists of 50 unit cells; each unit cell is composed of 6 , eight-node mean-quadrature tetrahedral elements. Lateral boundary conditions on the bar result in a planar, uniaxial strain wave. The simulation is based on a mass lumping of one-sixth of the total mass at each vertex nodal point and one-twelth of the total mass at each mid-face nodal point.

\subsection{Orthogonal Hourglass Control}

The mean stress-mean strain rate formulation considers only the linear part of the velocity field. The remaining portion of the nodal velocity field is the so-called hourglass field. Excitation of these modes may lead to severe, unresisted mesh distortion. A method for isolating the hourglass modes so that they may be treated independently of the rigid body and uniform strain modes is required. This is accomplished by developing an hourglass "gradient operator." With an hourglass gradient operator, hourglass strains can be computed from the element's relocity field. By introducing a "modulus," 


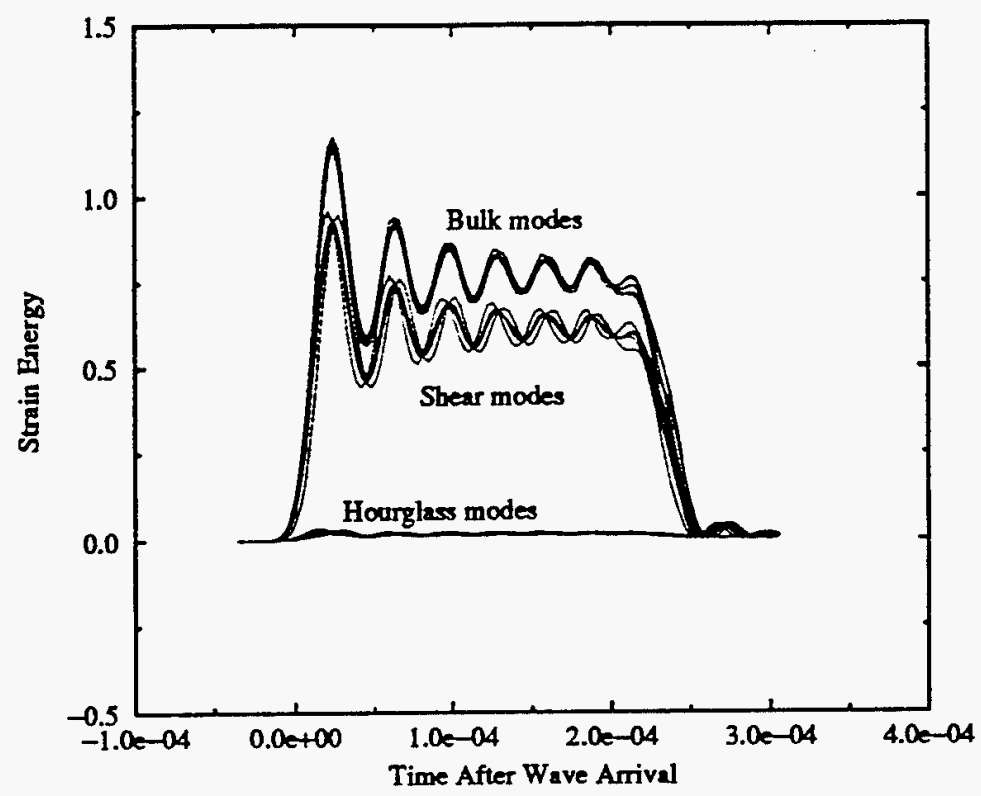

Figure 4: Bulk, shear and hourglass strain energy histories in a unit cell of 6 , eight-node mean-quadrature tetrahedra at the middle of a bar 50 units long. The simulation is based on a mass lumping of one-sixth of the total mass at each vertex nodal point and one-twelth of the total mass at each mid-face nodal point.

hourglass restoring forces can be generated and, thereby; prevent uncontrolled growth of the hourglass modes.

The linear velocity field on which the mean strain rates are based is given by

$$
v_{i}^{\mathrm{LIN}}=v_{i I}\left(\frac{1}{8} \Sigma^{I}+(1 / V)\left(x^{j}-\frac{1}{8} x_{J}^{j} \Sigma^{J}\right) B_{j}^{I}\right) .
$$

The hourglass velocity field $v_{i}^{\text {HG }}$ may be defined by removing the linear portion of the velocity field. Thus,

$$
v_{i}^{\mathrm{HG}}=v_{i}-v_{i}^{\mathrm{LIN}},
$$

or in terms of the nodal velocities,

$$
v_{i I}^{\mathrm{HG}}=v_{i I}-v_{i o} \Sigma_{I}-(1 / V)\left(x_{I}^{j}-x_{o}^{j} \Sigma_{I}\right) v_{i J} B_{j}^{J},
$$

where

$$
v_{i o}=\frac{1}{8} v_{i I} \Sigma^{I}, \text { and } x_{o}^{i}=\frac{1}{8} x_{I}^{i} \Sigma^{I}
$$


The hourglass velocity field, Equation 28 , is in the improper null space of the gradient operator $B_{i}^{I}$. The linear velocity field, Equation 26, spans 12 degrees of freedom: 3 rates of rigid body translation, 3 rates of rigid body rotation, and 6 uniform strain rates, which means that the hourglass subspace is the remaining 12 degrees of freedom.

Since we do not yet have an easy decomposition of the hourglass subspace into nonconstant bulk and shear modes, a rather direct approach is used. Thus, an hourglass strain rate $\dot{q}_{i I}$ is developed by operating on the hourglass velocities $v_{i I}^{\mathrm{HG}}$,

$$
\dot{q}_{i I}=v_{i I}^{\mathrm{HG}} / \delta,
$$

where $\delta$ is a generalized element dimension [Flanagan and Belytschko, 1984]. Using the hourglass projection operator $H_{I}^{J}$ implicit in Equation 28, the strain rate becomes

$$
\dot{q}_{i I}=\left(H_{I}^{J} / \delta\right) v_{i J} .
$$

To control the hourglass modes, generalized forces $Q^{i I}$ are defined that are conjugate to $\dot{q}_{i I}$, so that the work rate is given by

$$
v_{i I} f_{\mathrm{HIG}}^{i I}=I Q^{i l} \dot{q}_{i I} .
$$

Utilizing the projection operator $H_{I}{ }^{J}$, the contribution to the nodal forces due to hourglass resistance is given by

$$
f_{\mathrm{HG}}^{i I}=V^{\prime} Q^{i J} H_{J}^{I} / \delta .
$$

The hourglass restoring forces are calculated from

$$
\check{Q}^{i I}=\epsilon 2 \mu_{\tan } \delta^{i j} \delta^{I J} \dot{q}_{j J},
$$

where $2 \mu_{\tan }$ is the tangent shear stiffness obtained from the deviatoric constitutive behavior of the mean stress and mean strain state in the element, and $\epsilon$ is a scaling. The scaling $\epsilon$ assures the level of the hourglass restoring forces remains below that of the mean stress state.

Ideally the nonconstant hourglass shear strain rates would be assigned a stiffness derived from the tangent shear behavior and the nonconstant hourglass bulk strain rates would be assigned a stiffness derived from the tangent bulk behavior. Here the nonconstant bulk and shear modes are mixed, and the tangent shear modulus is used for all of the hourglass modes. The choice is arbitrary. The tangent modulus assures that the evolution of the hourglass restoring forces "parallels" that of the mean deviatoric stress state. 
The invariant time derivative of the generalized forces $Q^{i I}$ accounts for the finite rotations expected in the application of the eight-node tetrahedral element in analyzing transient dynamic phenomena. The derirative is given by

$$
\check{Q}^{i I}=\dot{Q}^{i I}-w_{j}^{i} Q^{j I},
$$

where $w_{i j}$ is the spin, $w_{i j} \equiv\left(v_{i, j}-v_{j, i}\right) / 2$.

The hourglass restoring forces $f_{\mathrm{HG}}^{i I}$ are added to those obtained from the divergence of the mean stress state so that the complete result is

$$
f^{i I}=\left(\bar{t}^{i j} B_{j}^{I}+V Q^{i J} H_{J}^{J} / \delta\right) .
$$

\section{Results}

\subsection{Irons Patch Test}

The first-order Irons patch test [Zienkiewicz and Taylor, 1989] provides a necessary condition an element must satisfy to insure convergence. In this test an irregular spatial discretization is required to determine if constant strain/stress states are reproduced. (Satisfying the first-order Irons patch test does not insure an "efficient" element. In particular it says nothing about the ability of a collection of elements to reproduce a linearly varying strain/stress result.)

Since a four-node, linear displacement tetrahedral mesh passes the firstorder Irons patch test, the purpose of revisiting the test for the current enriched eight-node tetrahedron is to insure that nothing has been lost in the proposed enrichment. The result of the first-order Irons patch test applied to an irregular mesh of enriched eight-node tetrahedra is a perfect reproduction of the constant strain/stress result in the interior of a cube subjected to an imposed linear displacement on the exterior, MacNeal and Ilarder [1985]. Implicit in this computation is the use of a linear elastic stress-strain material model.

For this element the imposition of a second-order Irons patch test is more significant since the element is a constant-strain (mean quadrature) element with hourglass control that was obtained by enriching a four-node linear displacement tetrahedron. Subjecting a collection of elements to a linear strain field will reveal the extent to which the well known locking of the four-node linear displacement tetrahedron has been ameliorated. 


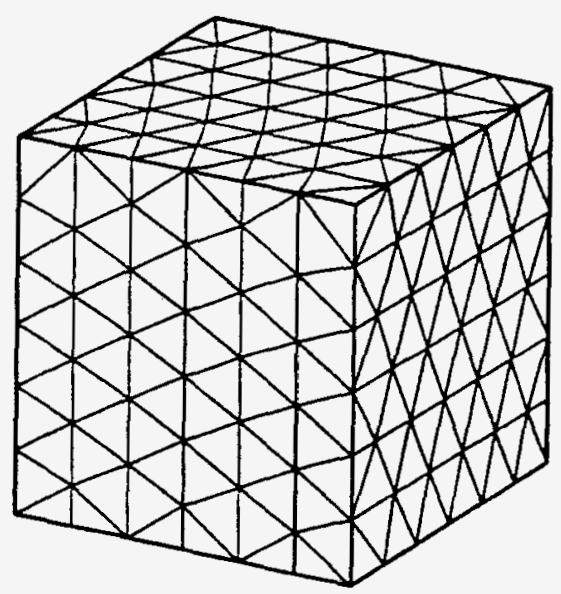

Figure 5: Displacement contours on a $10 \times 10 \times 10$ cube subjected to quadratic surface displacements in order to produce a body-force-free linear strain field for a second-order Irons patch test.

To produce a second-order Irons patch test, the cube of elements pictured in Figure 5 is subjected on the surface to the following prescribed quadratic displacement field,

$$
\begin{aligned}
u & =4 \times 10^{-6}\left(y^{2}+z^{2}-2 x^{2}+2 x y+2 x z+5 y z\right) \\
v & =4 \times 10^{-6}\left(x^{2}+z^{2}-2 y^{2}+2 x y+5 x z+2 y z\right) \\
w & =4 \times 10^{-6}\left(x^{2}+y^{2}-2 z^{2}+5 x y+2 x z+2 y z\right)
\end{aligned}
$$

for a range of Poisson's ratio $(0,0.1,0.2,0.3,0.4,0.499)$. This particular quadralic displacement field requires no auxiliary body forces to maintain the linearly varying strain/stress field and does not generate any volume change. Of particular interest is the amount of deviatoric strain energy and volumetric strain energy generated as a function of Poisson's ratio. (Linear elastic models for ductile metals typically have values for Poisson's ratio between 0.25 and 0.33 ; nearly incompressible materials are modeled with values of Poisson's ratio approaching 0.5.)

The results for both the four-node and the mean quadrature eight-node tetrahedral elements are displayed in Table 5, along with the results for the eight-node hexahedral element. As can be seen in Table 5 , the eightnode hexahedral elements exhibit no volumetric strain energy until values of Poisson's ratio approaching 0.5 are used, and then only small amounts 
Table 5:

Deviatoric and Volumetric Strain Energy Totals Obtained from a Second-Order Irons Patch Test.

\begin{tabular}{|c||cc|cc|cc|}
\hline $\begin{array}{c}\text { Poisson's } \\
\text { Ratio }\end{array}$ & \multicolumn{2}{|c|}{$\begin{array}{c}\text { Eight-Node } \\
\text { Hexahedron }\end{array}$} & \multicolumn{2}{c|}{$\begin{array}{c}\text { Four-Node } \\
\text { Tetrahedron }\end{array}$} & \multicolumn{2}{c|}{$\begin{array}{c}\text { Eight-Node } \\
\text { Tetrahedron }\end{array}$} \\
\hline 0.000 & 1146.8 & r'd-off & 1152.8 & 0.627 & 1134.6 & 0.456 \\
0.100 & 1042.5 & r'd-off & 1048.0 & 0.767 & 1031.7 & 0.379 \\
0.200 & 955.63 & r'd-off & 960.69 & 0.994 & 945.95 & 0.300 \\
0.300 & 882.12 & r'd-off & 886.84 & 1.429 & 873.43 & 0.222 \\
0.400 & 819.10 & r'd-off & 823.64 & 2.653 & 811.33 & 0.135 \\
0.499 & 765.00 & 0.100 & 770.36 & 233.5 & 758.11 & 0.020 \\
\hline
\end{tabular}

of parasitic volumetric strain energy are present. The standard four-node tetrahedral element exhibits small amounts of parasitic volumetric strain energy until values of Poisson's ratio of 0.5 are approached at which time unacceptably large values of parasitic volumetric strain energy are generated. The eight-node tetrahedral element proposed here exhibits small values of parasitic volumetric sirain energy until values of Poisson's ratio of 0.5 are approached at which time the parasitic volumetric strain energy begins to vanish. This surprising result can only bode well for elastic-plastic analyses where the deviatoric strain field is significantly larger than the volumetric strain field.

\subsection{Uniaxial-Strain Compression}

Of considerable practical importance is knowing the correct method of obtaining consistent nodal forces from an applied surface traction. Using the previously discussed procedure of putting nodal forces normal to each triangular facet subjected to pressure in the eight-node tetrahedron, the magnitudes of which are $\frac{1}{3} p A_{\text {facet }}$, produces the displacement contours shown in Figure 6.

IVith this method of computing equivalent nodal forces, the axial displacement varies linearly from the restrained face to the loaded face and is constant over the cross section. The stress within the interior of the cube is 


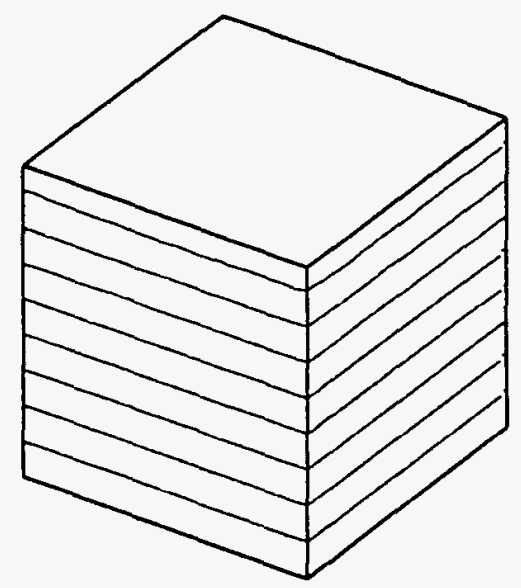

Figure 6: Axial displacement contours on a $10 \times 10 \times 10$ cube subjected to constant pressure on one face with the lateral surfaces constrained to produce a uniaxial-strain result.

constant. These results taken together confirm that consistent nodal forces have been obtained.

\subsection{Cylindrical Shear Wave}

Since the elements here pass a first-order Irons patch test, constant or nearly constant strain domains are not a severe test. However, wave fronts where the strain is changing rapidly from element to element will test the element's ability to avoid volumetric locking. A test for volumetric locking of a finite element mesh can be generated by subjecting a plane strain disk to an imposed torsional deformation on its inner radius while holding the outer radius fixed. A cylindrically divergent, pure transverse shear wave is generated. Any pressure in the solution domain is solely due to inherent numerical shortcomings in the finite element approximations for the gradient/divergence operator.

Three meshes are examined: (1) a hexahedral mesh in which each element block is a copy of the seven-element MacNeal and Harder [1985] first-order Irons patch test, (2) a classical four-node linear displacement tetrahedral mesh, and (3) a mean quadrature eight-node tetrahedral mesh, Figure $\bar{i}$.

This form of an irregular hexahedral mesh is used for two reasons: (1) a mesh that is nonaligned with the solution is obtained and is, therefore, comparable to the tetrahedral meshes which by nature are rarely aligned 
with the solution, and (2) comparable element totals are obtained for all three meshes.

In the elastic solution stress levels are on the order of 300,000 psi. To evaluate the amount of pressure generated relative to the effective stress (magnitude of the deviatoric stress), a Figure of Merit $\mathcal{F}(t)$ is computed at each time step by taking the ratio of the total bulk internal energy' to the total shear internal energy;

$$
\mathcal{F}(t) \equiv \frac{\int_{V} p e_{k}^{k} d v}{\int_{V} t^{m n} e_{m n} d v}
$$

The ideal value for the Figure of Merit $\mathcal{F}$ in the elastic case is zero, $\mathcal{F}(t) \equiv 0$. Figure 8 shows the Figure of Merit $\mathcal{F}$ for all three meshes during the time that the elastic shear wave propagates from the inner boundary to the outer boundary of the disk and returns to the inner boundary. While the Figure of Merit $\mathcal{F}$ for all three meshes is low, they stand approximately in the ratios of 20:10:1. The eight-node hexahedral mesh provides the best result being closest to zero, the four-node tetrahedral mesh remains the poorest performer having the largest Figure of Merit values, and the eight-node tetrahedral mesh lies between the other two Figure of Merit results.

Following the indications of the second-order Irons Patch Test results where the mean quadrature eight-node tetrahedron showed unexpected capabilities in the face of a nearly-incompressible elastic calculation, the cylindrical shear wave calculation was repeated with an isotropic, linear hardening elastic-plastic material representation (yield stress $=2.0 \times 10^{4} \mathrm{psi}$ and hardening modulus $=1.0 \times 10^{6} \mathrm{psi}$ ).

The ideal value for the Figure of Merit $\mathcal{F}$ in the elastic-plastic case is also zero, $\mathcal{F}(t) \equiv 0$. Figure 9 shows the Figure of Nerit $\mathcal{F}$ for all three meshes during the time that the elastic-plastic shear wave propagates from the inner boundary to the outer boundary of the disk and returns to the inner boundary. While the Figure of Merit $\mathcal{F}$ for all three meshes is low, they stand approximately in the ratios of $70: 1: 1$. For the elastic-plastic case the eight-node hexahedral and eight-node tetrahedral meshes both provide the best result being closest to zero; the four-node tetrahedral mesh remains the poorest performer having the largest Figure of Merit values. 
Table 6:

Taylor Bar Impact Results for OFHC Copper.

Initial Length $L_{0}=25.4 \mathrm{~mm}$; Initial Diameter $D=7.62 \mathrm{~mm}$

\begin{tabular}{|c||c|c|c|c|}
\hline $\begin{array}{c}\text { Final } \\
\text { Dimension }\end{array}$ & $\begin{array}{c}\text { Experimental } \\
\text { Measurement }\end{array}$ & $\begin{array}{c}\text { Johnson } \\
{[1988]}\end{array}$ & $\begin{array}{c}\text { Eight-Node } \\
\text { Hexahedron }\end{array}$ & $\begin{array}{c}\text { Eight-Node } \\
\text { Tetrahedron }\end{array}$ \\
\hline Length & $16.2 \mathrm{~mm}$ & $17.2 \mathrm{~mm}$ & $17.2 \mathrm{~mm}$ & $17.2 \mathrm{~mm}$ \\
Diameter at $0.2 L_{0}$ & $10.1 \mathrm{~mm}$ & - & $9.6 \mathrm{~mm}$ & $9.6 \mathrm{~mm}$ \\
Base Diameter & $13.5 \mathrm{~mm}$ & $14.6 \mathrm{~mm}$ & $14.4 \mathrm{~mm}$ & $14.1 \mathrm{~mm}$ \\
\hline
\end{tabular}

\subsection{Taylor Impact Tests}

Experimental results from Taylor impact tests proride data with which to make comparisons. The large plastic flows that occur to the cylindrical metal specimens used in the Taylor impact test provide exceptional mesh distortions thereby testing a range of geometric shapes for which the element geometry must provide reliable gradient and divergence calculations. Others have used Taylor impact experiments to test constitutive models as well. $c f$. , Johnson and Holmquist [1988]. Figure 10 shows the final calculated shape of what was originally a right circular cylinder of OFHC copper traveling 190 meters per second and impacting a flat, hardened steel target. To conduct the simulation, an implementation of the Johnson-Cook constitutive model was used, along with the following properties for OFHC copper: Young's modulus $E=124 \mathrm{GPa}$, Poisson's Ratio $\nu=0.34$, density $\rho=8960 \mathrm{~kg} / \mathrm{m}^{3}$, specific heat $C_{p}=383 \mathrm{~J} / \mathrm{kgK}$, melt temperature $T_{m}=1356 \mathrm{~K}$, room temperature $T_{r}=295 \mathrm{~K}$, the constant $A=90 \mathrm{MPa}$, the constant $B=292 \mathrm{Mpa}$, the exponent $n=0.31$, the constant $C=0.025$, and the exponent $m=1.09$, Johnson and Cook [1983].

Table 6 tabulates the experimental results, calculated results from Johnson [1988], calculated results based on an eight-node hexahedral finite element, and calculated results based on the mean-quadrature eight-node tet rahedral finite element. All of the calculated results are close to each other and stand in the same relationship to the experimental results. This outcome is typical for a simulation based on the Johnson-Cook constitutive model, Johnson and Cook [1983]. (An improved correlation with the experimentally observed results can be obtained with a Zerilli-Armstrong constitutive model, 
Johnson [1988].) For the purposes here it is clear that the mean-quadrature eight-node tetrahedron performs well in this simulation.

\section{Conclusions}

One is led to the conclusion that of the three finite elements examined, the mean-quadrature implementation of the eight-node hexahedron due to Flanagan and Belytschko [1981] remains numerically the more effective element. However, for simulations where the deviatoric strain field is significantly larger than the volumetric strain field (e.g., simulations based on nearlyincompressible elastic or elastic-plastic material behavior). the eight-node tetrahedron is an equally viable choice. To the extent that mesh generators can automatically fill arbitrary volumes smoothly and efficiently with tetrahedra, the eight-node tetrahedron proposed here is a satisfactory alternative to an eight-node hexahedral finite element and meshes requiring an inordinate amount of user intervention and direction to generate. The four-node linear displacement tetrahedron does not provide useful results in any case.

The use of mid-face nodes while leading to a large number of degrees of freedom does admit a compatible family of low-order mean-quadrature finite elements: an eight-node hexahedron, an eight-node pentahedron (a wedge with a mid-face node on each triangular end face), a 9-node Egyptian pyramid (mid-face nodes on each triangular face), and the eight-node tetrahedron presented here. 


\section{References}

Camacho, G. T., Jr., 1996. Computational Modeling of Impact Damage and Penetration of Brittle and Ductile Solids, Ph.D. Dissertatiou, Brown University, Providence, RI.

Flanagan, D. P., 1981. Numerical Techniques with One-Point Integrated Hexahedron and Quadrilateral Elements, Ph.D. Dissertation, Northwestern University, Evanston, IL.

Flanagan, D. P., and T. Belytschko, 1981. "A Lniform Strain Hexahedron and Quadrilateral with Orthogonal Hourglass Control," International Journal for Numerical Methods in Engineering, Vol. 17, pp. 679-706.

Flanagan, D. P. and T. Belytschko, 1984. "Eigenvalues and Stable Time Steps for the Uniform Strain Hexahedron and Quadrilateral," Journal of Applied Mechanics, Vol. 51, pp. 35-10.

Flanagan, D. P., and L. M. Taylor, 1989. PRONTO-3D, A ThreeDimensional Transient Solid Dynamic Program, Report Lo. SAND871912, Sandia National Laboratories, Albuquerque, NM.

Johnson, G. R., and W. H. Cook, 1983. "A Constitutive Model and Data for Metals Subjecled to Large Strains, Iligh Strain Rates, and IIigh Temperatures," Proceedings of the Seventh International Symposium on Ballistics, The Hague, The Netherlands, pp. 541-5+8 (April).

Johnson, G. R., and T. J. Holmquist, 1988. "Evaluation of Cylinder Impact Test Data for Constitutive Model Constants," Journal of Applied Physics, Vol. 64, No. 8, pp. 3901-3910 (October).

Johnson, G. R., 1988. "Implementation of Simplified Constitutive Models in Large Computer Codes," in Dynamic Constitutive/Failure Models, A. M. Rajendran and T. Nicholas, Eds.. Report No. AFVIALTR-88-4229, Air Force Systems Command, Wright-Patterson Air Force Base, Ohio 45433-6533.

Jones, R. E., 1964. "A Generalization of the Direct-Stiffness Method of Structural Analysis," AIA.4 Journal, Vol. 2, No. 5. 
Key, S. W., 1971. "A Specialization of Jones" Generalization of the Direct-Stiffness Method of Structural Analysis," AIA.A Journal. Vol. 9, No. 5 , pp. 98:-985.

Key, S. W. and C. C. Hoff, 1995. "An Improved Constant Mernbrane and Bending Stress Shell Element for Explicit Transient Dy'namics," Computer Methods in Applied Mechanics and Engineering. Vol. 124, pp. 33-4i.

Krieg, R. D. and S. W. Key, 1973. "Transient Shell Response by Numerical Time Integration," International Journal of . Vumerical Methods in Engincering. Vol. 7 , No. 3. pp. 273-286.

MacNeal, R. H., and R. L. Harder, 1985. "A Proposed Set Of Problems To Test Finite Element Accuracy;" Finite Elements In Design, Vol. 1, pp. 3-20, North-Holland Publishing.

Prager, W., 1967. "Variational Principles for Linear Elastostatics for Disconlinuous Displacement, Strains and Stresses," Recent Progress in Applied Mechanics: The F. Odquist Volume, pp. 463-4it, John Wiley \& Sons, New lork, NY.

Schmidt, E., 1907. "Zur Theorie der linearen und nichtlinearen Integralgleichungen. I. Teil: Entwicklung willkürlicher Funktionen nach Systemen vorgeschriebener," Mathematische Annalen, Vol. 63. pp. 433476; pp. $442-444$.

Zienkiewicz, CBE, FRS, O. C., and R. L. Taylor, 1989. The Finite Element Method, Fourth Edition, Vol. 1, McGraw-Hill Book Company' Europe, Berkshire, UK. 

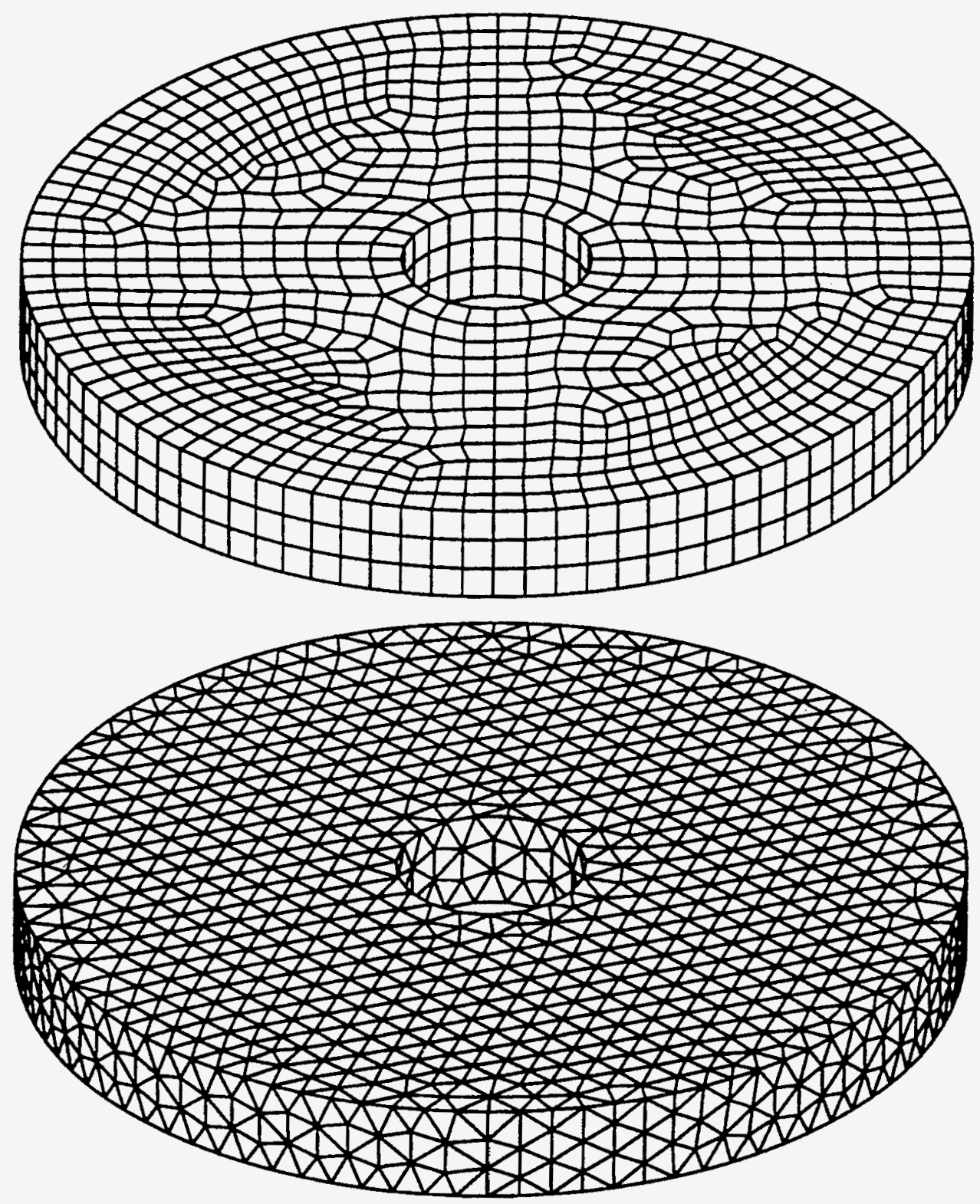

Figure $\bar{i}$ : Hexahedral and tetrahedral "flat washer" meshes (inner radius = 1 ; outer radius $=5$; thickness $=1$ ) used to model a cylindrically divergent, elastic, transverse shear wave (Young's modulus $=1.0 \times 10^{7} \mathrm{psi}$; Poisson's ratio $=$ of 0.25 ; density $\left.=2.61 \times 10^{-4} \mathrm{lbf}_{-} \mathrm{sec}^{2} / \mathrm{in}^{4}\right)$. The inner radius has an imposed twist; the outer radius is fixed. 


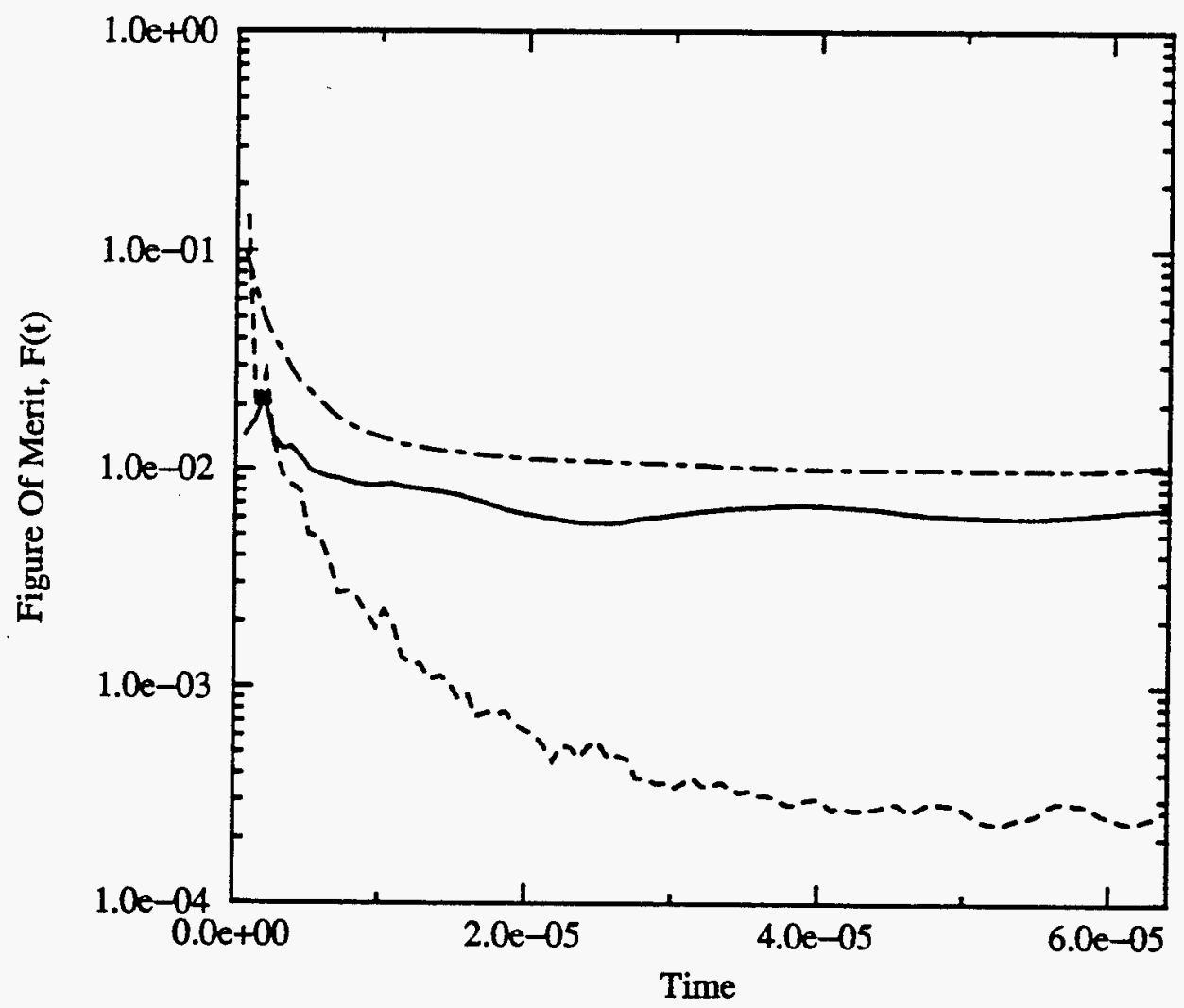

Figure 8: Values of $\mathcal{F}(t)$, Figure of Merit, for the time it takes the elastic transverse shear wave to travel from the inner radius to the outer radius and back for all three meshes. $(\mathcal{F}(t)$ for the eight-node tetrahedral mesh is the solid line. $\mathcal{F}(t)$ for the eight-node hexahedral mesh is the uniformly dashed line. $\mathcal{F}(t)$ for the four-node tetrahedral mesh is the short-long dashed line.) 


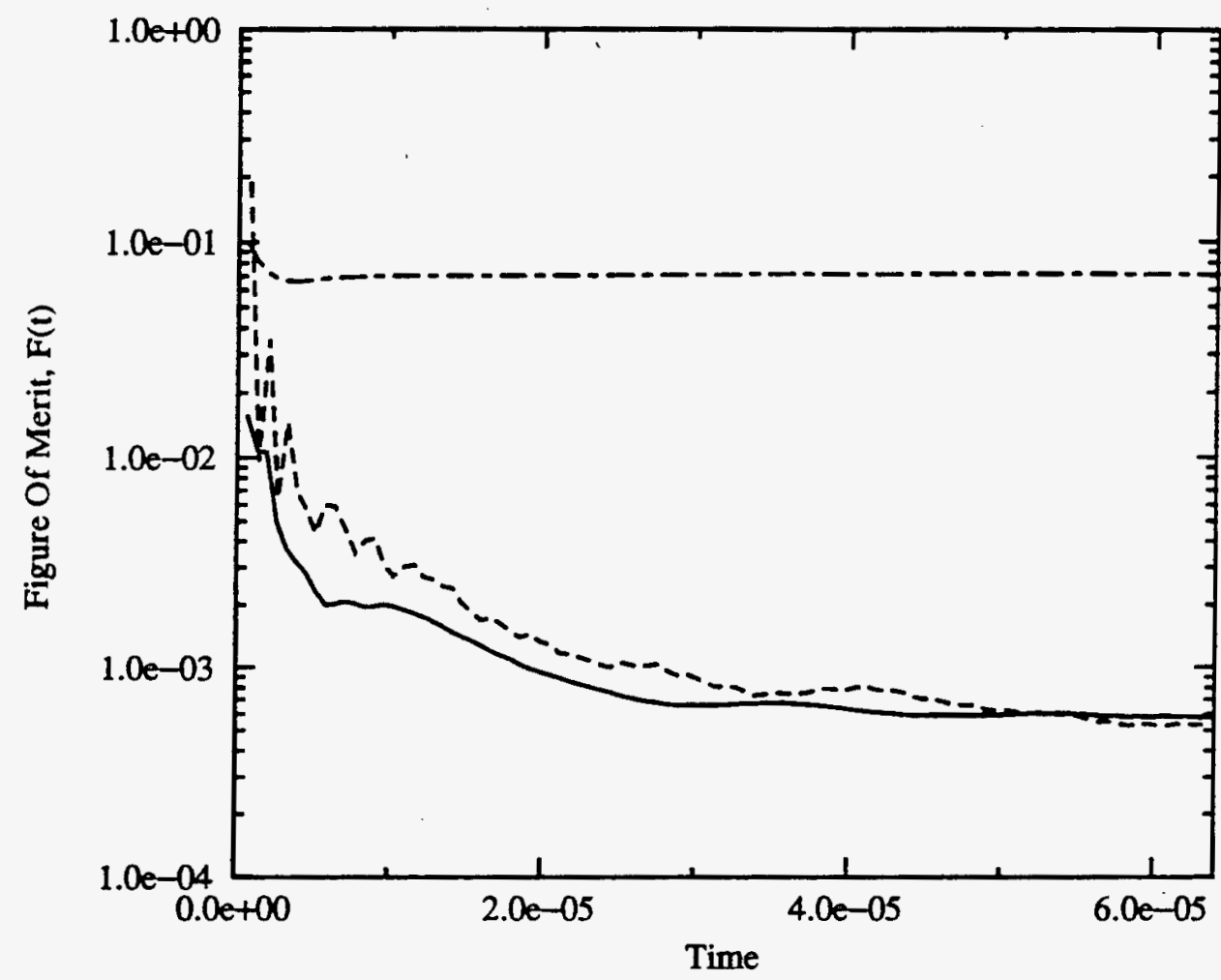

Figure 9: Values of $\mathcal{F}(t)$, Figure of Merit, for the time it takes the elastic-plastic transverse shear wave to travel from the inner radius to the outer radius and back for all three meshes. $(\mathcal{F}(t)$ for the eight-node tetrahedral mesh is the solid line. $\mathcal{F}(t)$ for the eight-node hexahedral mesh is the uniformly dashed line. $\mathcal{F}(t)$ for the four-node tetrahedral mesh is the short-long dashed line.) 


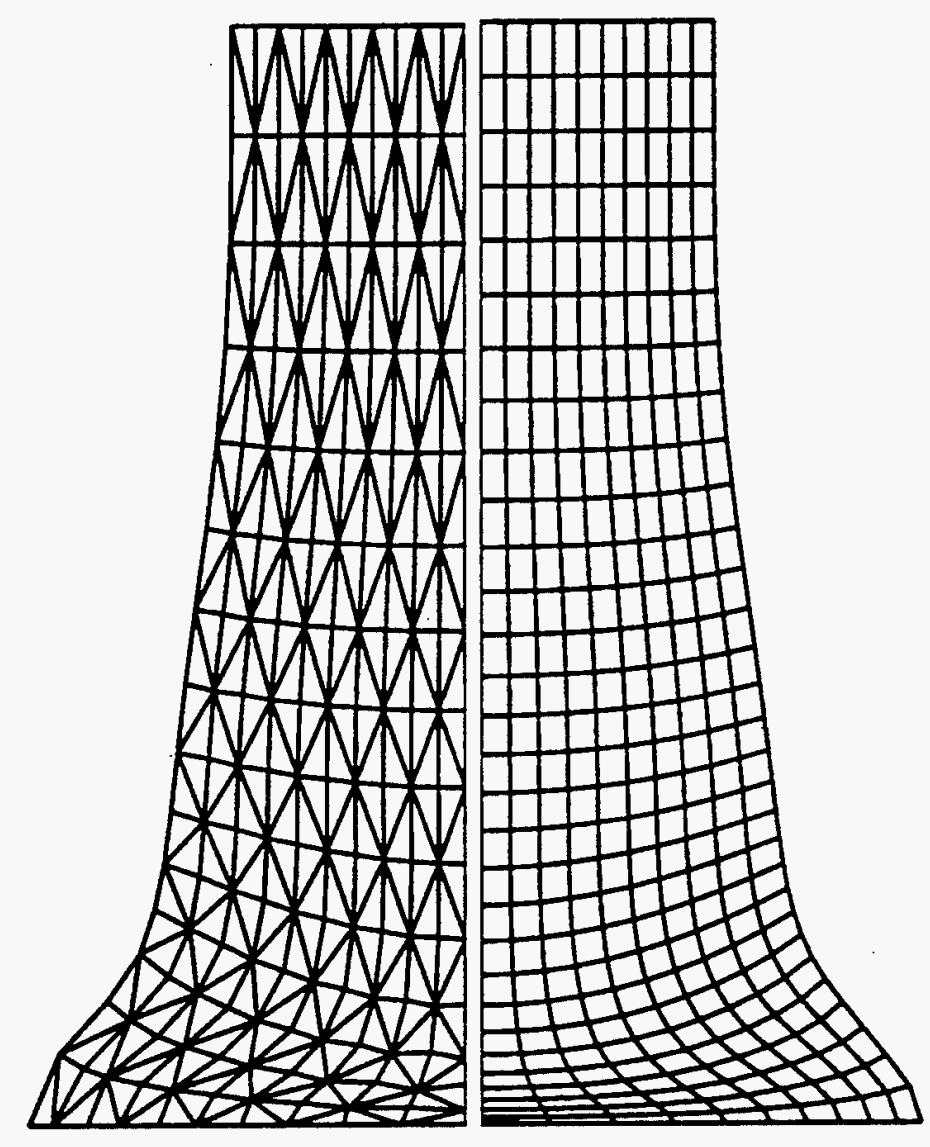

Figure 10: The final deformed shape of an OFHC copper rod impacted at $190 \mathrm{~m} / \mathrm{s}$ based on a simulation using a Johnson-Cook constitutive model. The left half of the figure is the result obtained using a mean-quadrature eight-node tetrahedral finite element mesh. The right lialf of the figure is the result obtained using a mean-quadrature eight-node hexahedral finite element mesh. (The mid-face nodal points in the tetrahedral mesh are not displayed.) 


\section{Internal Distribution:}

\begin{tabular}{|c|c|c|}
\hline 1 & MS 9042 & E.-P. Chen, 8742 \\
\hline 1 & MS 9042 & L. I. Weingarten, 8742 \\
\hline 1 & MS 9042 & L. E. Voelker, 8742 \\
\hline 1 & US 9405 & P. E. Nielan, 8743 \\
\hline 1 & NS 9042 & M. L. Chiesa, 8743 \\
\hline 1 & MS 9405 & J. F. Lathrop, 8743 \\
\hline 1 & .IS 0826 & D. K. Gartling, 9111 \\
\hline 1 & MS 0835 & R. J. Cochran, 9113 \\
\hline 1 & IIS 0443 & H. S. Morgan, 9117 \\
\hline 10 & IIS 0443 & M. L. Blanford, 9117 \\
\hline 10 & IIS 0443 & S. W. Key, 9117 \\
\hline 10 & .US 0443 & M. W. Heinstein, 911 T \\
\hline 10 & MS 0.43 & C. M. Stone, 9117 \\
\hline 1 & .VS 04.43 & J. R. Koteras, 9117 \\
\hline 10 & MS 0443 & F. J. Mello, 9117 \\
\hline 1 & MS 0443 & K. H. Brown, 9117 \\
\hline 1 & IIS $0+43$ & S. II. Attaway, 9117 \\
\hline 1 & IIS 0443 & J. Jung, 9117 \\
\hline 1 & MS 0443 & J. WI. Swegle, 9117 \\
\hline 1 & MS 0443 & M. R. Tabbara, 9117 \\
\hline 1 & MS 0443 & L. M. Taylor, $911 \bar{\imath}$ \\
\hline 1 & ISS 0819 & J. S. Peery 923I \\
\hline 10 & MS 0819 & K. G. Budge, 9231 \\
\hline 1 & MS 0819 & J. R. Weatherby, 9231 \\
\hline 1 & IIS 0439 & D. R. Martinez, 9234 \\
\hline 2 & IIS 0439 & C. R. Dohrmann, 9234 \\
\hline 1 & MS 0439 & D. J. Segalman, 9234 \\
\hline 1 & MS 9018 & Central Technical Files, 8940-2 \\
\hline 2 & MS 0899 & Technical Library, 4916 \\
\hline 2 & MS 0619 & $\begin{array}{l}\text { Review \& Approval Desk, } 12690 \\
\text { For DOE/OSTI }\end{array}$ \\
\hline
\end{tabular}




\section{External Distribution:}

Dr. Claus C. Hoff

MacNeal-Schwendler Corp.

815 Colorado Boulevard

Los Angeles, CA 90041-1777

Dr. Cees Florie

MacNeal-Schwendler Benelux B. V.

Groningenweg 6

2803 PV Gouda

The Netherlands

Dr. Dennis P. Flanagan

Ilibbitt, Karlsson \& Sorenson, Inc.

1080 Main Street

Pawtucket, RI 02860--4847

Mr. Roger Keene

Hibbitt, Karlsson \& Sorenson (UK), Ltd.

Genesis Center

Science Park South, Birchwood

Warington, Cheshire WA37BII

United Kingdom

Prof. Ted Belytschko

Theoretical and Applied Mechanics

Northwestern University

2145 Sheridan Road

Evanston, IL 60208-3020

Prof. K. C. Park

Center for Space Structures \& Controls

Campus Box 429

University of Colorado

Boulder, CO 80309

Dr. Jeffrey Weiss

Orthopedic Biomechanics Institute

5848 South 300 East

Salt Lake City, UT 84107 
Report Number (14)

SAND- $-98-0756$

\begin{tabular}{|c|c|}
\hline Publ. Date (11) & 199803 \\
\hline Sponsor Code (18) & $D O E / D P$ \\
\hline UC Cate & UC-721 DOETER \\
\hline
\end{tabular}

